\title{
Novel Fracture Site Targeting Drug Improves Fracture Healing with Pain Relief: Preclinical Evaluation of MAK123
}

\author{
Abduallah Elsayed ${ }^{1}$, Jeffery Nielsen ${ }^{4,5,6}$, Natalie Taylor $^{2}$, Mohammed Juboori ${ }^{2}$, Caio de Andrade \\ Staut $^{1}$, Vincent Alentado ${ }^{3}$, Nicholas Hux ${ }^{1}$, Christopher Dalloul ${ }^{1}$, Samuel Zike ${ }^{1}$, Nikhil Tewari ${ }^{1}$, \\ Murad Nazzal ${ }^{1}$, Hanisha Battina ${ }^{1}$, Alex Brinker ${ }^{1}$, Mustafah Shaikh ${ }^{1}$, Sarah Myers ${ }^{1}$, Rachel \\ Blosser $^{1}$, Ushashi Dadwal ${ }^{1}$, Mary Niedrauer ${ }^{4}$, Stewart Low ${ }^{4}$, Philip Low ${ }^{4,5,6}$, Jiliang Li ${ }^{7}$, Fletcher \\ A. White ${ }^{2,8}$, Melissa A. Kacena ${ }^{1,8}$ \\ Departments of ${ }^{1}$ Orthopaedic Surgery, ${ }^{2}$ Anesthesia, and ${ }^{3}$ Neurological Surgery, Indiana University School \\ of Medicine, IN \\ ${ }^{4}$ Novosteo Inc., West Lafayette, IN \\ Departments of ${ }^{5}$ Medicinal Chemistry and Molecular Pharmacology and ${ }^{6}$ Chemistry, Purdue University, \\ West Lafayette, IN \\ ${ }^{7}$ Department of Biology, Indiana University Purdue University Indianapolis, Indianapolis, IN \\ ${ }^{8}$ Richard L. Roudebush VA Medical Center, Indianapolis, IN
}

Non-union bone fracture occurs in $5-10 \%$ of fracture injuries. Interventions include surgery with local implantation of autograft, allograft, demineralized bone matrix, and/or bone morphogenetic proteins. These types of fracture injuries are also accompanied by acute and chronic pain states. In most instances, opioids are provided to injured patients during and after surgery. With the opioid crisis, identifying new analgesic therapies that could reduce or eliminate opioid use, while also improving bone healing is important. Here we show the ability of a novel compound, MAK123, to both enhance bone healing and reduce pain behavior in a surgically induced femoral fracture mouse model. Briefly, 20 male C57BL/6 mice underwent a surgically induced femoral fracture and then were treated with $0,2,6$, or $20 \mathrm{mg} / \mathrm{kg}, 3 X /$ week for the 3 week study duration. Weekly X-rays were used to examine healing progression. Prior to euthanasia, mice underwent behavioral testing to measure evoked pain behaviors. Upon euthanasia, ex vivo $\mu C T$ imaging and analysis was completed to assess fracture callus size and composition. While all doses of MAK123 tested resulted in improved healing, the $6 \mathrm{mg} / \mathrm{kg}$ dose resulted in accelerated bone healing and a significant increase in mineralized callus volume $(p<0.05)$. Similarly, while all doses of MAK123 reduced evoked responses to tactile stimulus as demonstrated by increased paw withdrawal thresholds, $6 \mathrm{mg} / \mathrm{kg}$ of MAK123 resulted in a more robust and significant improvement $(p<0.05)$. We postulate that optimization of the dosing schedule/concentration could further improve both bone healing and behavioral measures thought to represent pain in rodents. That said, these promising pre-clinical data warrant further evaluation as MAK123 may prove to be a unique tool for orthopaedic surgery usage whereby it could both improve bone healing and reduce clinical pain, improving overall patient outcomes. 